\title{
CONTRIBUCIÓN DE LAS NORMAS CIOMS 2002 AL DESARROLLO BIOTECNOLÓGICO. LA CORRESPONSABILIZACIÓN DEL CIENTÍFICO
}

\author{
ALEXANDRE BOTA ARQUÉ*
}

\begin{abstract}
La revolución biotecnológica afronta grandes retos, entre ellos la participación social, la bioseguridad y la protección tanto del germoplasma como del conocimiento ancestral que de él se derivan. Para afrontar estos dilemas se plantea la necesidad de buscar una corresponsabilización de todos los actores del desarrollo biotecnológico. En este artículo se realiza un correlato partiendo de la reflexión ya iniciada por la comunidad médica con sus pautas éticas internacionales para la investigación biomédica en seres humanos, CIOMS, versus la biotecnología para buscar una corresponsabilización de los tecnocientíficos, biotecnólogos en sus investigaciones con la pretensión utópica de buscar un desarrollo biotecnológico mas respetuoso con el hombre y con su entorno y que dé respuesta a los problemas que la biotecnología está generando.
\end{abstract}

Key terms: Bioética, Biotecnología, Empoderamiento.

En el presente trabajo se formula una propuesta de correponsabilización ética derivada de los términos creados por la racionalidad científica: Biodiversidad y Bioética. Es una propuesta sencilla que pretende extender las conclusiones de los acuerdos internacionales sobre biodiversidad y acceso a los recursos genéticos a los diferentes grupos sociales, pero enfocada especialmente versus la creación de un marco de correponsabilización de los investigadores aprendiendo de las experiencias históricas como la del Consejo de Organizaciones Internacionales de la Ciencia Médica CIOMS, con sus Pautas Éticas Internacionales para la Investigación y Experimentación Biomédica en Seres Humanos (1), elaboradas en colaboración con la Organización Mundial de la Salud.

\section{BIODIVERSIDAD}

La biodiversidad es una creación de la racionalidad científica. Es un término que va más allá de una definición etimológica, es un término polimórfico y transversal. Es una palabra que pretende englobar la multifactorialidad de la realidad viva en la que constantemente el hombre se relaciona y se integra. El término biodiversidad pretende comprender la diversidad biológica, la diversidad cultural, la diversidad de todo lo vivo, pero no como sistemas autónomos, sino como sistemas interrelacionados, y esto sucede porque los distintos ámbitos del conocimiento han ido convergiendo desde diferentes ópticas en el estudio de esa realidad de la cual el hombre es parte consustancial. Asumir la palabra biodiversidad implica, por lo tanto, asumir una gran amplitud de miras y enterrar de una vez por todas el reduccionismo científico. Trabajar con material genético proveniente de la biodiversidad implica tener muy presentes las implicaciones sociales, simbólicas, económicas de la biodiversidad.

\section{BIOÉTICA}

La bioética al superar el ámbito clínico y abrirse a problemas más globales se imbrinca íntimamente con la biodiversidad. La Bioética no existe y no

\footnotetext{
* Centro Interdisciplinario de Estudios en Bioética.

Universidad de Chile, Providencia 1017, Piso 7, Providencia, Chile. Miembro del Instituto de Tecnoética de Barcelona

Telephone: (56 2) 678 2274. Fax (56 2) 678 2254. E-mail: botaa@chi.ops-oms.org
} 
puede existir como un cuerpo normativo o de conocimientos, existe únicamente el interés bioético de los biólogos, los sociólogos, los científicos, los carpinteros o los habitantes del planeta. Lo que la Bioética pretende es trascender el sentido etimológico de los términos en cada racionalidad y superarlos. Pretende hacer dialogar al físico con el sociólogo y a su vez con la trabajadora, partiendo de su propia realidad, pero buscando un acuerdo. La bioética trasciende el reduccionismo científico y la verdad tecnocrática para hallar una praxis transformadora y es desde esta concepción que se plantea la reflexión, ya que se pretende integrar a los máximos elementos en el proceso de correponsabilización de las decisiones para hacer reales los múltiples marcos jurídicos creados.

En el presente artículo se inicia una reflexión que tendrá que ser proseguida por los investigadores con el objetivo de generar un marco de correponsabilización de los investigadores en los procesos de desarrollo de nuevos productos biotecnológicos. Este proceso de corresponsabilización ha de ir parejo de adopciones legales como normativas de acceso a recursos genéticos, que ya existen en algunos países latinoamericanos, normativas de bioseguridad, etc. Con el propósito de no dejar toda la responsabilidad a la ética al investigador.

El empoderamiento de la comunidad científica facilitará la aplicación real de los marcos jurídicos. Entendiendo empoderamiento como la habilidad del científico para ganar comprensión y control sobre las fuerzas personales, sociales, económicas y políticas, en orden a actuar para mejorar la situación (2). Un primer paso en pos de este empoderamiento sería la recontextualización de muestras biológicas, separadas actualmente de la naturaleza, a muestras que forman parte de la naturaleza, esto obligaría a entender que la descontextualización y la modelización (3) son herramienta del método científico útil para el discurrir dentro de su ámbito, pero generadoras de conflictos cuando estas interaccionan con la realidad. La materia viva no es una realidad concreta separada del mundo, sino que forma parte consustancial del mismo. Finalmente los científicos, tendrían que asumir en su yo más íntimo que el antiguo ideal de la neutralidad de la ciencia está muerto y que actualmente ellos están situados en la era de la tecnociencia (4).
Es imperioso para el científico tomar consciencia afectiva y discursiva de que hay múltiples intereses detrás de sus investigaciones. El científico racionalmente se da cuenta de estos intereses, pero este darse cuenta no es transformado en discurso ni en praxis, es asumido y escondido debajo de múltiples pretextos. Todo profesional relacionado con la biotecnología sabe que existen unos intereses económicos muy importantes detrás de su desarrollo. Legítimos o no, no pretendemos cuestionarlos. El científico ha de tomar consciencia que no está situado en la ciencia y en el discurso científico, sino que se encuentra situado en un espacio tecnocientífico, donde la tecnificación de la ciencia o la cientificación de la técnica (5) ha polarizado enormemente la realidad que él percibe. El investigador, el científico ahora es un tecnocientífico.

En este darse cuenta de los diferentes intereses detrás de la tecnociencia, el Investigador ha de recuperar su responsabilidad, asumiendo la no neutralidad de su trabajo, permitiendo el accionar social sobre su trabajo y correponsabilizándose de él. El tecnocientífico, debido a su gran especialización, abandonó esa supuesta visión amplia, surgida del enfrentamiento con la naturaleza y actualmente circunscribe su realidad a la del laboratorio, pretendiendo, a pesar de todo, continuar poseyendo esa áurea de buscador de conocimiento, cuando en realidad no es más que un artesano altamente cualificado.

El investigador, como cualquier persona, sufre las presiones de los deseos, estas pueden fomentar su espíritu inquieto o pueden dar salida a unos intereses que en el discurso racional no aceptaría o encontraría poco éticos. Para el tecnocientífico el mejor postor no es únicamente la rentabilidad económica. El mejor postor puede ser publicar en cierto tipo de revistas, participar en ciertas instituciones, conseguir recursos financieros, etc. El tecnocientífico intentará enfocar sus investigaciones siguiendo una serie de líneas de investigaciones previamente fijadas por intereses de diversa índole: económicos, políticos, tecnocientíficos, sanitarios, que han sido previamente definidos por el sector empresarial, con la participación de los políticos y los científicos próximos al poder y sin tener presentes los intereses de la sociedad en una visión amplia y global, y no generándose espacios de democracia participativa. Los 
tecnocientíficos y los políticos se creen justos representantes de los intereses sociales, pero sería necesario un poco de honestidad en la comunidad científica para reconocer los intereses propios.

¿A quién le interesará trabajar en nuevas semillas híbridas si lo que está de moda y genera prestigio son los OvGM? La novedad es un condicionante muy importante, unida íntimamente o a la de progreso indefinido. La novedad tecnológica puede no ser la respuesta social y sustentable más adecuada (6). Pero la novedad es lo que le permitirá al tecnocientífico responder a las necesidades de su entorno, publicaciones, recursos para finalmente ser reconocido.

\section{CORRESPONSABILIZACIÓN DEL INVESTIGADOR}

El avance tecnocientífico genera continuamente nuevos retos que se convierten en problemas éticos de difícil solución debido principalmente a que no existen muchos referentes por los cuales discurrir, siendo interesante aprender de los aciertos y los errores pasados de las disciplinas cercanas. Uno de los errores del pasado en las discusiones sobre las investigaciones médicas con sujetos humanos, fue pensar que toda la responsabilidad ética consistía en el buen diseño del experimento y en la calidad moral de los investigadores. Se creyó que los científicos se autorregularían, pero a la vista de lo que sucedió fue necesario crear otras estructuras que supervisasen y elaborasen directrices. Como el Código de Nuremberg (1947) (7) elaborado como respuesta las atrocidades nazis, o la Declaración de Helsinki (1964, última revisión 2000) (8), conjuntamente con otras declaraciones como el Pacto sobre derechos civiles y políticos de las Naciones Unidas del 1976, donde en su artículo 7, estipula que "nadie será sometido a tortura ni a pena o tratos crueles inhumanos o degradantes. En especial, nadie será sometido sin su libre consentimiento a experimentos médicos o científicos"(9).

Frente a la necesidad de plasmar las intenciones reflejadas en esas declaraciones, en 1981 la Asociación Médica Mundial empezó a formular posibles pautas orientativas que se plasmaron en las Pautas Internacionales Propuestas para la Investigación Biomédica de 1982, las cuales debido al enorme éxito, se convirtieron al ser modificadas en Pautas CIOMS en 1993, siendo revisadas y ampliadas en el año 2002.
Estas pautas no pretenden ser marcos jurídicos cerrados sino flexibles ya que pretenden ir adaptándose al ritmo cambiante de la tecnociencia, para solucionar los recovecos que podrían quedar en unas pautas no impositivas, en la revisión de 2002 a diferencia de la de 1993 (10), se busca la corresponsabilización de los diferentes estamentos implicados en el desarrollo de la investigación con seres humanos. Y de su desarrollo, sobre todo de las modificaciones aparecidas en el año 2002, la biotecnología puede ahorrarse muchas discusiones y avanzar velozmente creando espacios de reflexión. En la actualidad la biotecnología afronta un elevado rechazo social, el cual no se puede reducir únicamente a cuestiones de percepción pública, sino que afectan mucho más profundamente a toda la sociedad.

Los puntos que podrían ser altamente interesantes para una reflexión social sobre la biotecnología en este artículo son parcialmente inspirados en las normas CIOMS 2002. No se pretende iniciar la discusión sobre la necesidad de extender los derechos de los hombres a los otros vivientes de la tierra, sino que se pretende hacer una reflexión a partir del reconocimiento de que el hombre no se limita a lo físico y mental. La interacción del hombre con la naturaleza es una expresión del hombre, el sujeto no puede entenderse solo como individuo, organismo individual, sino como sujeto que conforma un sistema La concepción de hombre no puede limitarse al hombre físico. En varios fragmentos las normas CIOMS hablan de la salud física y mental. A razón de lo expuesto anteriormente es necesaria la extensión de los conceptos de salud física y mental a los términos social y ecológico. Situaciones de nocividad ambiental repercutirán a la larga en un deterioro de la salud física y deterioros de la situación social conducirán inevitablemente a un deterioro de la salud mental (11). El desarrollo de la biotecnología afectará directamente a la estructuración social, redistribución de los trabajos, materias primas que podrán ser producidas en fábricas biotecnológicas modificándose las relaciones económicas entre los países, también se verá afectada la biodiversidad, introducción de cultivos masivos, contaminación genética, alteración de los ecosistemas, así como la concepción más interna de lo que es la naturaleza. Es por esto necesario abrir espacios de reflexión colectivos y no únicamente 
técnicos y el juego de utilizar las normas CIOMS como esqueleto puede ser enriquecedor.

En la siguiente tabla I se esquematiza la idea inspiradora de las diferentes pautas de las normas CIOMS y cuál podría ser su traslación a algunas supuestas normas para investigadores, bioprospectores, empresas, etc.

De los posibles ítemes que pretende abordar las normas CIOMS, solo nos centraremos en algunos puntos muy concretos dejando de lado para posteriores trabajos puntos tan relevantes, como la obtención del consentimiento social de acceso a los recursos genéticos, la estructura de los comités de bioseguridad y biodiversidad, la transferencia de tecnología, la simbolización de los objetos naturales.

Para enmarcar y poder interpretar la filosofía de las pautas, introduciremos fragmentos de las aclaraciones a las pautas CIOMS de 2002, que aportan pistas para su traslación a la reflexión sobre el desarrollo biotecnológico.

La Pauta 10 se refiere a la investigación con personas de recursos limitados que se podría trasladar en el caso de la biotecnología a la

TABLA I

Correlato de las Pautas CIOMS a Pautas para la biotecnología

\begin{tabular}{ll}
\hline Investigación con sujetos humanos & Investigación biotecnológica \\
\hline Comités de ética & Comités de bioseguridad y biodiversidad \\
Pauta 2,3 & Configuración de los comités. No únicamente criterios técnicos \\
Configuración de los comités. & Acuerdo Social \\
Consentimiento Informado & Consentimiento Informado Social. \\
Consentimiento Informado Individual & Acuerdo público \\
Acuerdo privado, confidencialidad &
\end{tabular}

Pauta 5

Obtención del consentimiento:

Pauta 6

Obligaciones de los patrocinadores y

los investigadores con relación al sujeto

Pauta 8

Beneficios y riesgos de la participación

\section{Pauta 9}

Especiales limitaciones y riesgos de la investigación cuando esta afecta a personas incapaces de dar su consentimiento

\section{Pauta 10}

Investigación en poblaciones o comunidades con recursos limitados

\section{Pauta 12}

Distribución equitativa de perjuicios y beneficios en la selección de grupos de sujetos en la investigación

\section{Pauta 19}

Derecho de los sujetos de recibir en caso de ayuda tratamiento y compensación

\section{Pauta 20}

Fortalecimiento de la capacidad de revisión ética y científica de la investigación biomédica
Obtención del acuerdo de acceso a los recursos genéticos. Reparto de beneficios

Obligaciones de los patrocinadores e investigadores con relación a la comunidad y al entorno

Beneficios y riesgos ambientales y sociales de la investigación.

Alteración de estructuras sociales, modificación de hábitos, pérdida de biodiversidad, etc.

Limitaciones especiales cuando se trabaja con ecosistemas muy delicados y con culturas que poseen otra simbolización de la naturaleza

Investigación en países o comunidades en vías de desarrollo

Distribución equitativa de las fases de desarrollo del producto biotecnológico

Derecho de los sujetos y los ecosistemas de recibir ayuda en caso de contaminación

Fortalecimiento de la capacidad de conocer y valorar capitalistamente lo que se posee y fortalecimiento de la capacidad en bioseguridad. Capacitación de los investigadores en otras concepciones sobre la naturaleza

Obligaciones éticas de las empresas para revertir los beneficios que han obtenido de la explotación del germpolasma 
investigación en países en vías de desarrollo, bioprospecciones. En las aclaraciones dicen que "el requisito ético de 'respuesta adecuada' solo puede ser cumplido si las intervenciones exitosas u otras clases de beneficios en salud se ponen a disposición de la población". Si se trasladasen a la bioprospección muchas de las investigaciones no serían éticamente realizables. "Si el conocimiento obtenido a partir de la investigación en un país es usado primariamente para beneficio de las poblaciones que pueden costear el producto probado, la investigación puede correctamente ser caracterizada como explotadora y, por lo tanto, no ética". Esto legitima la utilización del término expoliación para la gran mayoría de bioprospección. "Si un medicamento a investigar se ha demostrado que es beneficioso, el patrocinador debería continuar proveyéndolo a los sujetos después de la conclusión del estudio, y pendiente de su aprobación por una autoridad reguladora de medicamentos" Si se halla una propiedad medicinal, agrícola, etc., interesante, las empresas deberían facilitar el acceso a los nativos por un tiempo determinado o indefinido. Si la investigación no busca el desarrollo de un nuevo producto sino el incremento del conocimiento se debe estar seguro de que el conocimiento usado será utilizado en beneficio de la comunidad que ha convivido con ese ecosistema, como dicen las normas CIOMS: "Debe haber seguridad, sin embargo, de que el conocimiento científico desarrollado será usado para beneficio de la población". Las bioprospecciones son el talón de Aquiles del desarrollo biotecnológico. Es entonces necesario que los científicos asuman su parte de responsabilidad, la naturaleza, no puede ser expropiada, aunque sean organismos que no hayan interaccionado con el hombre, organismos marítimos, zonas vírgenes, aguas termales, etc. Es por esto que se convierte en imprescindible que los científicos contribuyan a la elucidación de caminos que den respuesta a estos conflictos, uno de ellos podría ser la trazabilidad a origen de las muestras biológicas. $\mathrm{Si}$ se conoce la procedencia de un gen, de una cDNA, geográficamente, de la especie, se podrá afrontar mejor el reparto de beneficios.

El comentario a la Pauta 12 -distribución equitativa de cargas y beneficios en la selección de grupos de sujetos en la investigación- afirma que "La igualdad requiere que ningún grupo o clase de personas cargue con más de lo que es justo en compartir los perjuicios de participar en la investigación. Del mismo modo, no debería privarse a nadie de su justa parte en los beneficios de la investigación”. El desarrollo biotecnológico está acrecentando las diferencias entre los países proveedores de germoplasma y los países que poseen la tecnología, lo que pareciera legítimo para los sujetos no se extrapola a la sociedad, agrupación de sujetos. El comentario a dicha norma no promulga la distribución igualitaria lo que dice es "la distribución desigual no debe ser no equitativa". No se pretende que la distribución de los beneficios tenga que ser para todos igual, sino que esta ha de ser equitativa. Una distribución igualitaria podría alterar enormemente comunidades tradicionales. Lo que sucede en la actualidad es que el desarrollo biotecnológico no está favoreciendo a todas las comunidades que poseen o han conservado la biodiversidad planetaria, el continente latinoamericano es la gran reserva de la biodiversidad global, pero la implantación de industria biotecnológica en el continente es insignificante y las patentes de productos biotecnológicos de pertenencia latinoamericana solicitadas en la USPTO, oficina de patentes americana, son no significativos (12). Lo cual refleja la gran asimetría del desarrollo biotecnológico entre países que poseen la biodiversidad y los países industrializados que poseen la tecnología y las patentes.

El comentario a la Pauta 19 sobre los derechos de los sujetos dañados por tratamientos es muy importante ya que la biotecnología en sus ámbitos de investigación y aplicación puede colisionar y dañar tanto a los sujetos individualmente, socialmente y a los ecosistemas. La Pauta afirma que "los investigadores deberían garantizar que los sujetos de investigación que sufren algún perjuicio como resultado de su participación tienen derecho a tratamiento médico gratis por tal perjuicio y a apoyo económico o de otro tipo que pueda compensarlos equitativamente por cualquier menoscabo, discapacidad o minusvalía resultante". Los campesinos o los ecosistemas salvajes que sufran contaminación por productos biotecnológicos al amparo de esta posición podrían exigir responsabilidades a las empresas y exigir el tratamiento de descontaminación a las empresas biotecnológicas. Es importante 
profundizar el último comentario a la norma 19: "No debe pedirse a los sujetos la renuncia al derecho de compensación" ya que parece ser que las empresas biotecnológicas no quieren asumir esta responsabilidad y exigen a los ciudadanos que los eximan de esta responsabilidad. La empresa biotecnológica tendrá que asumir la misma responsabilidad con sus productos que las farmacéuticas con los efectos tóxicos o colaterales de los fármacos.

Con relación a la Pauta 20 que se refiere al fortalecimiento de la capacidad de revisión ética y científica de la investigación biomédica, posee una traslación muy clara en el campo biotecnológico. Muchas naciones han sufrido bioprospecciones legales e ilegales sin conocer previamente lo que poseían. Además, muchos países en vías de desarrollo han implementado productos biotecnológicos, semillas, sin tener la capacitación necesaria en materia de bioseguridad para hacerlo. ¿Cómo es que si en temas médicos hay una tan elevada sensibilidad y precaución en temas sociales y ecológicos este principio de precaución se diluye?

Las pautas que podrían tener una traslación a la biotecnología en el fondo son todas y muchas otras que tendrían que elaborarse, ya que la biotecnología afecta mucho más ampliamente a la sociedad, la biodiversidad, en todos sus ámbitos y de maneras diferentes.

\section{CONCLUSIÓN}

Pretender la implantación de unas normas como las Pautas CIOMS en la investigación biotecnológica puede resultar altamente complicado, primeramente por la amplitud de organismos con los que se trabaja, ya que no todos generan para la humanidad los mismos conflictos éticos. La relación entre un humano y una lacto bacilus es sustancial y formalmente diferente de la que establecen los humanos con los primates, así que el conflicto ético es diferente. Además, como la biotecnología en el laboratorio trabaja a nivel molecular, las dificultades se acrecientan, inconmensurabilidad de paradigmas. Es como pretender interpretar la temperatura, que es una magnitud macroscópica, a nivel microscópico. Lo cual dificulta más el acuerdo y el diálogo, pero aunque el esfuerzo pueda resultar vano, el camino y la reflexión abierta resulta por sí misma suficientemente enriquecedora para humanizar el desarrollo de la biotecnología, posiblemente será imposible llegar a un acuerdo formal, pero el esfuerzo para entender los otros puntos de vista puede ser altamente productivo, aunque totalmente intangible.

Otro punto de conflicto es que las modificaciones de los organismos biotecnológicos solo son detectables en la cultura tecnocientífica disminuyéndose la autonomía de los individuos de otras culturas al relacionarse con estos organismos, de lo cual se desprende que puede ser necesaria la formación de los científicos en otras simbolizaciones de la naturaleza. Asumir que los organismos naturales poseen diferentes simbolizaciones culturales, religiosas, pueden permitir al científico comprender otras posiciones.

Lo que sí que se podría aprovechar a la luz de lo anunciado en la página sobre la correponsabilización del científico, es el aprovechar el espíritu de las normas CIOMS y realizar formalmente propuestas como las que las normas CIOMS poseen en su Apéndice I donde se podría exigir que se especificase claramente lo que el científico tiene que conocer o saber y reflexionar antes de embarcarse en un proyecto de investigación. El tecnocientífico tendría que reflexionar sobre las relaciones que se establecen entre investigadores, patrocinadores y otros sujetos, tendría que reflexionar sobre las consecuencias tanto positivas como negativas de su proyecto, también se tendría que buscar un compromiso ético de los editores y los patrocinadores, cuando con las muestras biológicas de ARN; ADN; tejido, células, organismo, etc., se pretendiese obtener algún beneficio económico, sería bueno que pudiera haber una trazabilidad hasta el origen para ver a quién han de revertir parte de los beneficios. Las muestras proceden de algún punto del planeta, la generación espontánea no existe. Se ha de conseguir el método de hacer trazables las muestras hasta su origen y trazable la información básica que de ella se posee. Si esta trazabilidad se esclareciese, como se pretende hacer con los productos alimentarios procedentes de transgénicos, los conflictos éticos disminuirían enormemente y se dejaría de culpabilizar a los investigadores. Los investigadores son los más interesados en esclarecer su situación y su responsabilidad, pero pueden mostrarse temerosos a trascender su ámbito de 
conocimiento y una posible solución puede emerger al socializar la problemática. Los dilemas éticos generados por la biotecnología son una buena oportunidad para recuperar una visión más amplia de la realidad y la ciencia.

\section{AGRADECIMIENTOS}

Este trabajo fue realizado en el transcurso de un proyecto de Investigación del Centro Interdisciplinario de Bioética de la Universidad de Chile ejecutado durante una estadía en el Programa Regional de Bioética de la Organización Panamericana de la Salud, fruto de las reflexiones surgidas en el trabajo de traducción al español de las normas CIOMS.

\section{BIBLIOGRAFÍA}

1. CIOMS (2002) Pautas Éticas Internacionales para la Investigación Biomédica en Seres Humanos. Chile: Ed Programa Regional de Bioética OPS/OMS
2. CANAVAL GE (1999) Propiedades psicométricas de una escala para medir percepción del empoderamiento comunitario. Colombia Médica 30 http:// colombiamedica. univalle.edu.co/Vol30No2/ empoderamiento.html

3. LADRIER JEAN (1977) El reto de la racionalidad. Salamanca: Ed Sígueme, Cap III

4. RODRÍGUEZ-ALCÁZAR FJ, MEDINA-DOMÉNECH RM, SÁNCHEZ-CAZORLA JA (1997) Ciencia, Tecnología y Sociedad. Contribuciones para una cultura de la paz. Instituto de la Paz y los Conflictos de la Universidad de Granada. Cap VII

5. HOTTOIS G (1985) El Paradigma Bioético, Barcelona: Ed Anthropos

6. ALTIERI M (2003) Dimensiones éticas de la crítica agroecológica a la biotecnología agrícola. Acta Bioethica XI: 1

7. Tribunal Internacional de Nuremberg (1947) Código de Nuremberg

8. Asociación Médica Mundial (2000) Declaración de Helsinki (versión 2000)

9. Naciones Unidas (1976) Acuerdo sobre Derechos Civiles y Políticos de las Naciones Unidas, Artículo 7

10. CIOMS (1993) Pautas éticas Internacionales para la Investigación y Experimentación Biomédica en Seres Humanos. Traducido por ICMER. Santiago de Chile

11. LOLAS F (2001) Normatividad fisiológica y nocividad ambiental. Acta Bioethica VII, 2: 205-212

12. BOTA A (2003) La biotecnología en Latinoamérica, espacios de participación social. Acta Bioethica XI: 115-135 